\title{
BLAST analysis of Sars-Cov-2 deliniates the functionality of the virus's proteins
}

\author{
Diana Maria Popescu* and Eliza Marghidanu* (students at Tudor Vianu National High School of \\ Computer Science) \\ The National Institute of Research and Development for Biological Sciences, Bucharest, Romania \\ (Internship)
}

\begin{abstract}
:
In light of recent events that have been happening across the globe, I and my colleague decided to bring light upon the main characteristics of the new coronavirus outbreak from the Wuhan province in China, in December 2019. We first compared this SARSCov-2 to the old SARS-Cov-1 that appeared in 2003 and found a striking similarity between their genomes and therefore as we showed in our papers they also share some properties. Then we identified the main proteins that form the Covid-19 and in order to find new characteristics of this virus. This paper has informing purposes as in these dark times the best way to cooperate with the ongoing threat is to learn more about it.
\end{abstract}

\section{Introduction:}

Coronaviruses are a large family of viruses that generally determine mild to moderate upperrespiratory tract illnesses, such as the common cold, in people. For example, there have been three times in the 21 st-century coronavirus outbreaks that have emerged from animal hosts to cause critical disease and international transmission concerns.

There are a multitude of coronaviruses, most of which circulate among animals including pigs, camels, bats and cats. Occasionally, those kinds of viruses jump to humans -named a spillover event, which we will address later- and can determine the disease. Seven kinds of coronaviruses are known to cause human illnesses, four of each have moderate intensity: viruses 229E, NL63, OC43 and HKU1. (1) The other three can cause serious outcomes in people, and those diseases are SARS, also known as severe acute respiratory syndrome that emerged at the end of 2002 and vanished in 2004; MERS or Middle East respiratory syndrome, which appeared in 2012 and is still circulating in camels (2). Finally, the COVID19 outbreak (that is caused by the coronavirus known as SARS-CoV_2) started in December 2019 in China and at the moment global efforts are performed to avoid the spreading of this deadly virus.

Furthermore, the coronaviruses are RNA viruses that have positive-sense (3) genomes that can act as mRNA and be used to synthesize proteins without the help of a complementary RNA intermediate, making this viruses immediatelly translated by the host cell. (4) Because of this, these viruses do not need to have an RNA polymerase packaged into the virion. The overall structure of all coronaviruses is composed of spike $(\mathrm{S})$, envelope $(\mathrm{E})$, membrane $(\mathrm{M})$, and nucleocapsid (N). (5) (6) 


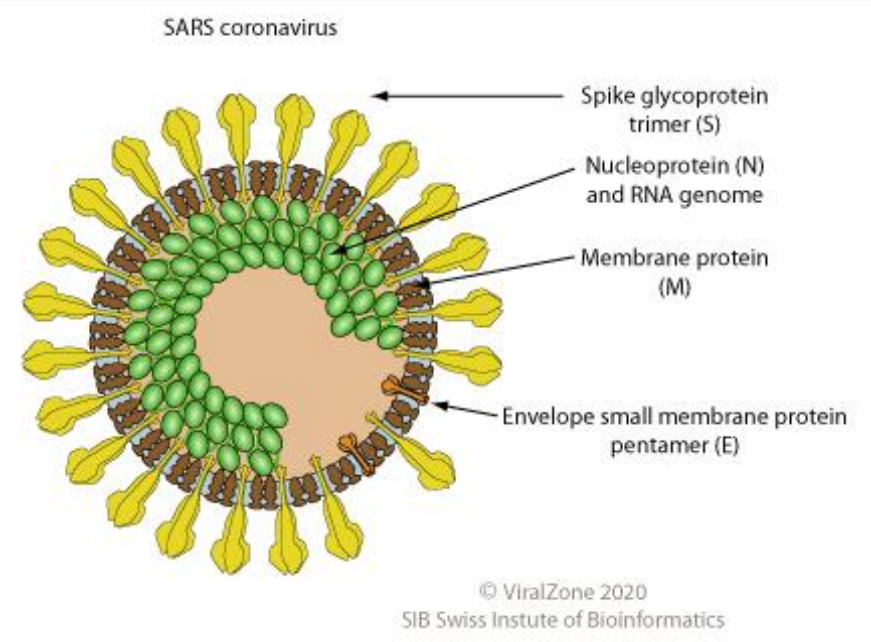

Figure 1 - The SARS's Viron Structure (7)

\section{Overview on the outbreak from 2002-2004 of the SARS in China}

On 11 February 2003, the Chinese Ministry of Health notified the World Health Organization (WHO) of an outbreak of peculiar pneumonia that likely emerged in Guangdong Province, China, in November 2002. (8) During late February to early March 2003, clusters of atypical pneumonia emerged in Vietnam, Hong Kong, Canada, and Singapore, infecting approximately 8459 patients and resulting in more than 800 deaths (9). The overall mortality rate was approximately $14-15 \%$, ranging from less that one percent in persons of 24 years to more than $50 \%$ in persons with more than 65 years. (10)

One of the first known cases was a doctor from Guangdong who traveled to Hong Kong in order to visit his family for about one week after the onset of some symptoms. During his visit, he infected other people that stayed in the same hotel as the doctor. (11) (12)

\section{Probable cases of SARS by week of onset Worldwide* $(n=5,910), 1$ November 2002 - 10 July 2003}

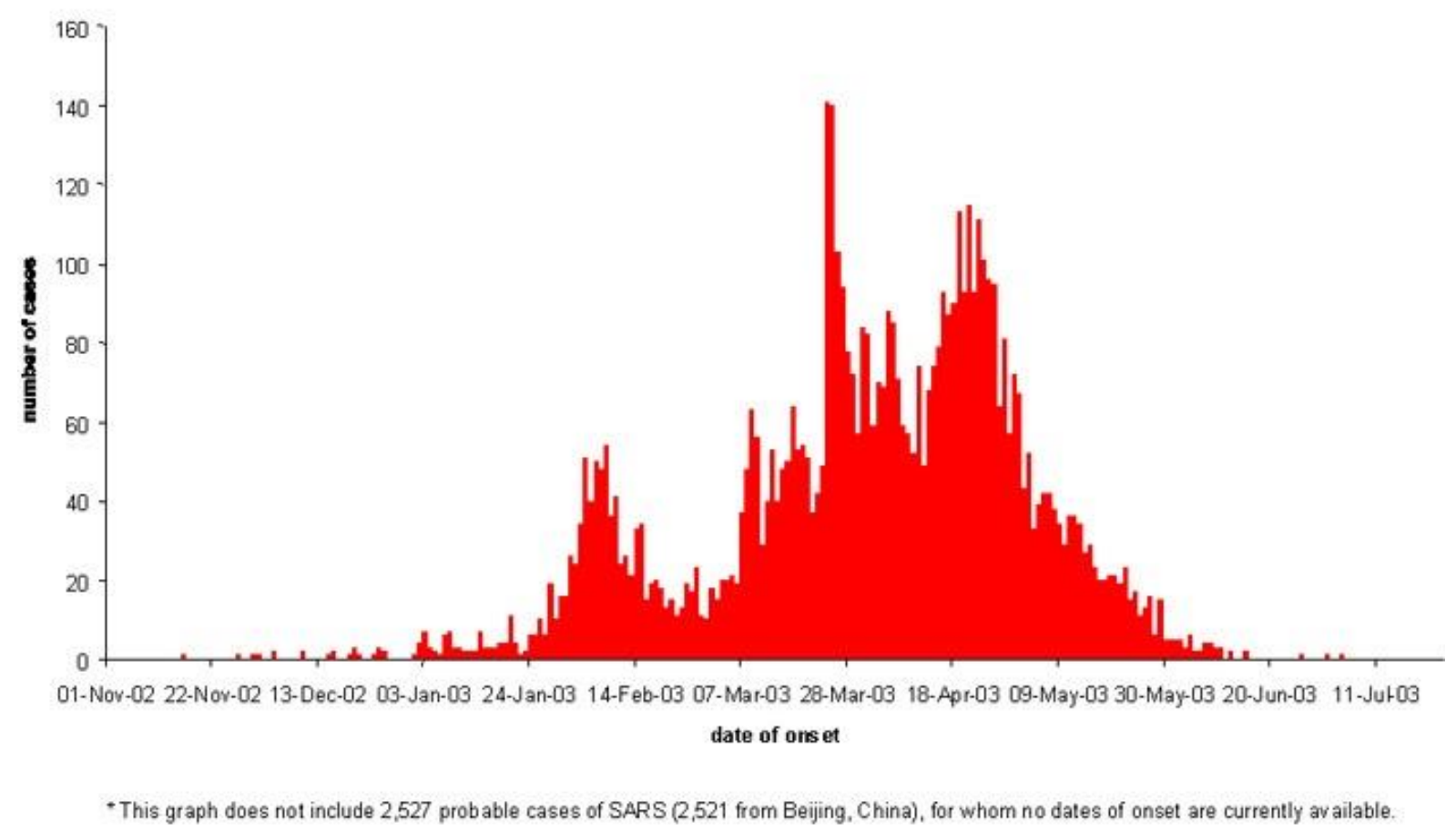

Figure 2 - Probable cases of SARS by week of onset worldwide, 1 November 2002 - 10 July 2003 (9) 


\section{Similarities and differences between 2019-nCoV and SARS}

We took the complete structure of the SARS-CoV_2 genome and tried to find its closest match in order to see its characteristics and it was no surprise when we discovered that the genome structure of the 2003 coronavirus was the closest to the actual virus. Overall, by using the BLAST program, we found out that the genome of $2019-\mathrm{nCoV}$ has $89 \%$ nucleotide identity with bat SARS-like-CoVZXC21 and 82\% with that of human SARS-CoV. (13) Therefore, we tried to conceive a list of similarities of the viruses and also of differences, that can be used for a better understanding of what the world is fighting against.

\subsection{Spreading}

Both the SARS and the COVID-19 are spread in small droplets of saliva coughed or sneezed into the air by an infected person. If someone else breathes in the droplets, they can become infected. The viruses can also be spread indirectly if an infected person touches surfaces such as door handles with unwashed hands and a healthy person touches the same surface. (14) Some of the most common carriers are bats, although they do not typically transmit coronaviruses directly to humans. Instead, the transmission might occur via an "intermediary" animal, which will usually - though not always - be a domestic one. There has been a total of 8,096 clinically diagnosed cases of SARS worldwide, with 774 deaths and regions affected, according to the WHO data on July 31st, 2003. Most cases were in Asia, Europe, and America. According to the latest data on May 9th, 2020, there has been a total of 3,822,382 clinically diagnosed cases of COVID-19 worldwide, with 263,658 deaths. (15)

\subsection{Symptoms}

The clinical symptoms of 2019-nCoV infection are similar to those of SARS-CoV. Most patients present fever, dry cough, dyspnea, and bilateral ground-glass opacities on chest CT scans. However, patients with $2019-\mathrm{nCoV}$ infection rarely have obvious upper respiratory signs and symptoms (such as snot, sneezing, or sore throat), indicating that the virus primarily infects the lower respiratory tract. In addition, about $20-25 \%$ of $2019-\mathrm{nCoV}$ patients experience intestinal symptoms and signs (such as diarrhea), similar to SARS-CoV (16). In severe 2019-nCoV infection cases, the symptoms include acute respiratory distress syndrome, septic shock, metabolic acidosis, and bleeding and coagulation dysfunction. It is worth noting that severe and critically ill patients may have moderate to low fever during the course of the disease, even without obvious fever. Furthermore, like SARS-CoV, 2019-nCoV infections induce the production of high levels of cytokines. (17) (5) (18)The epidemic of 2019-nCoV bears some similarities to SARS-CoV. The outbreaks of the two viruses occurred at about the same time during the year, and they were quite stable in the environment, especially in airconditioned spaces, owing to lower ambient temperatures and lower humidity. However, SARS-CoV had an aberrant trait in that the "viral load" in upper respiratory tract secretions was low in the first 5 days of illness, then increased progressively and peaked early in the second week. Consequently, the transmission rate was relatively low in the first days of illness, providing an opportunity for case detection and isolation to interrupt transmission. On the contrary, for 2019-nCoV, the incubation lasts an average of 10 days (in a reported range of 2-14 days) from infection to symptoms surfacing. Even worse, 2019-nCoV is able to 
spread from one person to another even before any actual clinical manifestations, leading to "extremely challenging" conditions for detecting and isolating potential patients, which makes it more difficult to control the epidemic. (19)

\subsection{Structural differences}

By using the UniProt and BLAST databases we found out that one SARS-CoV accessory protein, known as $8 \mathrm{a}$, is absent in the new virus. Other accessory proteins vary in length. In 2019-nCoV, 8b is 37 amino acids longer than in SARS-CoV while $3 \mathrm{~b}$ is shorter by 132 amino acids. Instead, some 2019-nCoV proteins are more similar to those of bat SARS-like coronaviruses, while accessory proteins $3 \mathrm{a}$ and $8 \mathrm{~b}$ are most similar to the SARS-CoVs. However, the external subdomain of Spike's receptor-binding domain of 2019-nCoV shares only a $40 \%$ amino acid identity with other SARS-related coronaviruses. While the 2019nCoV was closer to bat-SL-CoVZC45 and bat-SL-CoVZXC21 at the whole-genome level, the receptor-binding domain of COVID-19 fell within lineage $\mathrm{B}$ and was closer to that of SARSCoV. (16)

\subsection{Current therapies}

There are currently no approved antiviral treatments or vaccines for human coronavirus infections. The development of safe, stable vaccines is a huge challenge, and vaccines would ideally be broad-spectrum, so the research and development of new drugs is a very long process. In such a sudden epidemic, scientists were not able to carry out new drug development by the traditional principles. Recently drug screening found that nelfinavir has potential antiviral activity against 2019-nCoV. In addition, pitavastatin, perampanel, and praziquantel might also have moderate activities against 2019-nCoV (20). Based on previous studies, an anti-HIV drug named Kaletra may have a therapeutic effect on SARS and MERS, which is composed of two protease inhibitors, ritonavir, and lopinavir. More recently, Kaletra was also recommended to treat Wuhan pneumonia by the National Health Commission of the People's Republic of China. (5) Patients with SARS or MERS have used several drugs including ribavirin, interferon, lopinavir-ritonavir, and corticosteroids, but the efficacy of certain drugs is still controversial. Other antiviral drugs, like FAD-approved drugs including ribavirin, penciclovir, nitrazine, nalfa musta, chloroquine, and the two broad-spectrum antiviral drugs redexivir (GS-5734) and favivir (T-705), are being evaluated by measuring the effects of these compounds on cytotoxicity, virus yield and infection rate of 2019-nCoV. Recent results showed that remdesivir and chloroquine are effective in controlling 2019-nCoV infection in vitro and may be evaluated in human patients with 2019-nCoV disease. Remdesivir has recently been recognized as a promising antiviral drug against multiple RNA viruses (including SARS/MERS-CoV) in cultured cells and in mouse and non-human primate (NHP) models (16). Currently, remdesivir for the treatment of Ebola virus infection is in clinical research. It is promising that these compounds can treat 2019-nCoV infections. Moreover, the fifth edition of infection prevention and control (IPC) guidance announced that severe and critically ill patients could be treated with recovery plasma. (20) Also, scientists investigate the possibility of treating the infected patients with Triazavirin, which is a guanine nucleotide originally developed in Russia that has shown efficacy against influenza A and B, including the H5N1 strain. (21) 


\section{Characteristics of the SARS-Cov_2 genome described from its proteins}

We discovered that 10 major proteins compose the SARS-Cov_2 genome and each of them conveys specific characteristics.

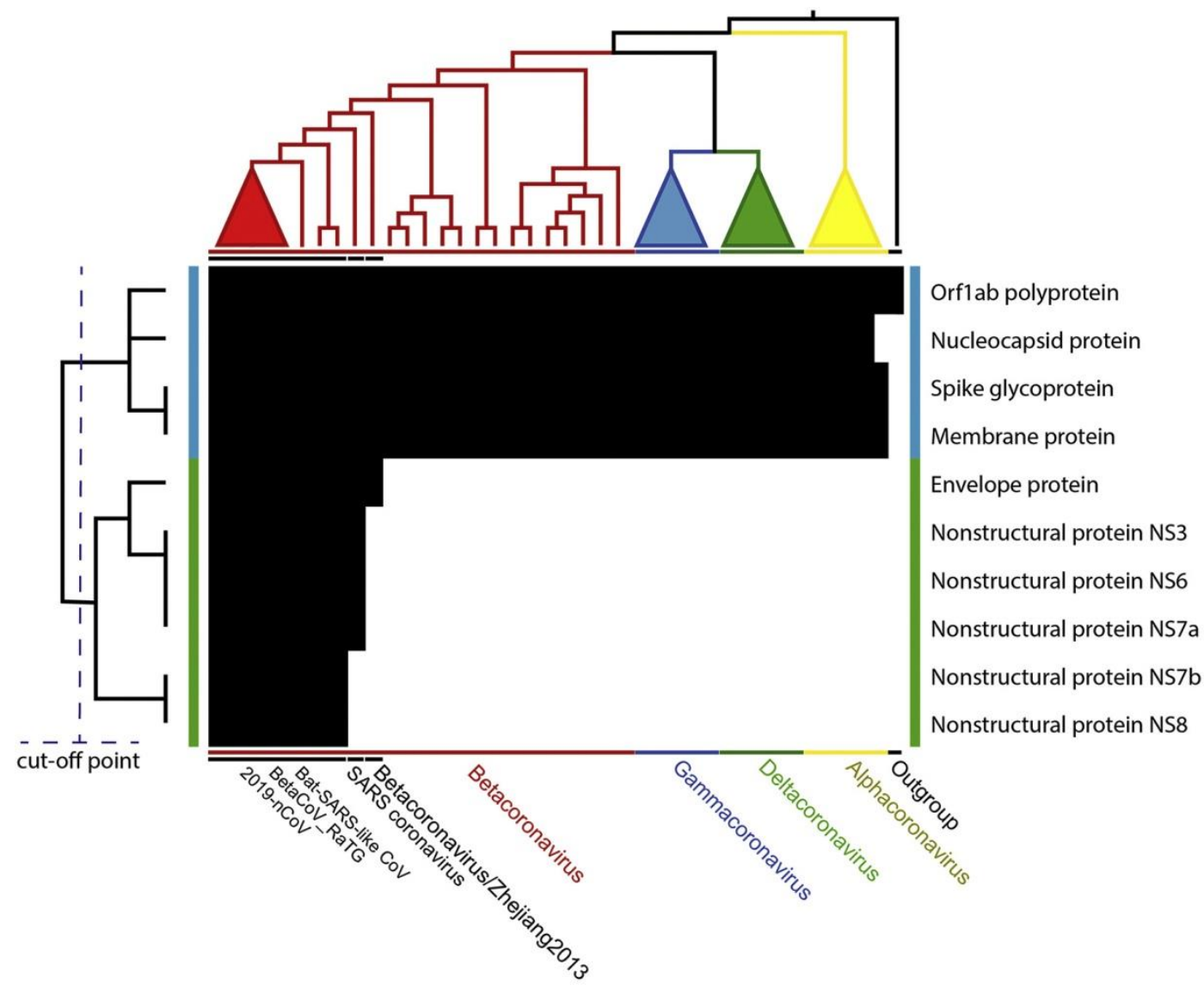

Figure 2 - Phylogenetic profiling of the annotated 2019-nCoV proteins combined with the maximum likelihood cladogram of the Orthocoronavirinae subfamily with Okavirus as the outgroup. (22) The heatmap shows the binary matrix of the similar proteins of 2019-nCov against other species in the set of data; black and white suggesting the presence and absence, respectively. The vertical tree is a phylogenetic profiling tree made from a binary matrix of the presence and absence of homolog proteins. It has two clades, indicated by blue and green bars. The horizontal tree is the cladogram of the maximum likelihood tree with a collapsed clade of $2019-\mathrm{nCoV}$. (22) 


\subsection{ORF1ab Polyprotein}

The replicase enzyme is displayed as two polyproteins (ORF1a and ORF1ab), which are prepared into 12 nonstructural proteins by three viral proteases (23). This ORF1ab polyprotein includes the nsps 1-3 proteins. This area of ORF1ab is the most important factor among coronaviruses (24). Many researchers found the relationship between ORFs with COVID-19 i.e.8782C $>\mathrm{T}(\mathrm{ORF} 1 \mathrm{ab})$ and $28144 \mathrm{~T}>\mathrm{C}(\mathrm{ORF})$ are available among genomic databases (25) (26). Hence, it will be clinically significant to break down the biological function of the particular protein ORF1ab in SARS-CoV-2.

The protein's biological processes are mainly induction by virus of host autophagy(meaning that this protein allows the virus to activate or increase frequency rate or extent of autophagy in the host) (27), viral genome replication (processes that are involved directly in viral genome replication, including viral nucleotide metabolism) (28) and viral protein processing that consists in the process of protein maturation achieved by the cleavage of peptide bond located within a viral protein (29). All these biological functions suggest that the SARSCov_2 spreads quickly as it multiplicates easily.

By running BLAST on this particular protein we discovered various other proteins that resemble more or less with orflab polyprotein. From that multitude we selected:

1. non-structural polyprotein $1 \mathrm{ab}$ [Bat SARS-like coronavirus]-that has a $95.55 \%$ identity

2. nonstructural polyprotein pp1ab [Severe acute respiratory syndrome-related (5) coronavirus]- that has a $86.05 \%$ identity (5)

3. orf1ab polyprotein [SARS coronavirus ExoN1]- that has a $86.02 \%$ identity

Both the non-structural polyprotein 1ab [Bat SARS-like coronavirus] and orf1ab polyprotein [SARS coronavirus ExoN1] have as biological processes (30) methylation (plays an important role in mRNA stability ,processing, transport and protein translation) (31) and transcription, DNA templated (which is a cellular synthesis of RNA on a template of DNA).

All three of them have as biological processes (5) induction by virus of host authopagy (which means that the virus activates or increases the frequency of authopagy in the host), viral genome replication and viral protein processing.

In addition, all three proteins can be found in the host's perinuclear region or in the host's membrane or in the multi-pass membrane protein. (5)

\subsection{ORF8 Protein}

Orf8 protein of SARS-CoV-2 doesn't contain a known useful motif or region. A total motif VLVVL (amino corrosive 75-79) has been found in SARS-CoV orf8b which was appeared to trigger intracellular stress pathways and enact NOD-like receptor family pyrin region containing-3 (NLRP3) ( (32)). Moreover, multiple arrangements with different coronavirus ORF8 sequences propose that L84 related to 28144T $>$ C (L84S) isn't preserved ( (33)). Thusly, it will be critical to examine the biological function of the particular protein (orf8) in SARS-CoV-2.

The main function of this protein of Human SARS coronavirus (SARS-CoV) (Severe acute respiratory syndrome coronavirus) is to form spikes at the surface of the virion envelope as they are essential for the initial attachment to heparan sulfate moieties of the host cell surface proteoglycans. It is also involved in the fusion of viral and cellular membranes that lead to the 
entry of the virus into the the host cell. (33) After this initial binding to its host receptors, the fusion machinery composed of $\mathrm{gB}$ and the heterodimer $\mathrm{gH} / \mathrm{gL}$ meditates the membrane fusion. Then, the ORF8 protein may be involved in the fusion between the virion envelope and the outer membrane during the virion egress. (34)

This protein has the following main biological processes: viral entry into host cell ( the process occurs after the viral attachment by a virus or viral nucleic acid that breaks the plasma membrane or cell envelope and enter the host cell; after this process, the viral nucleic acid is released into the host cell cytoplasm but sometimes the virus attachment results in the virion being moved by the host cell to another location (35), therefore the SARS-Cov_2 can be taken also from objects) and virion attachment to host cell (which represents the process by which a virion protein binds to molecules on the cellular surface or host cell surface projection) (36)

We tried to find some similar proteins to the ORF8 protein found in SARS Cov_2 by running BLAST and we found a multitude of alike proteins, most of them belonging being related to the previous coronaviruses. Therefore, we selcted the following:

1. nonstructural protein NS8 [Bat coronavirus RaTG13], that has a 95.04\% identity with the ORF8 protein, is specific for 2019-nCoV and its closely related species, BatCoV RaTG13 and Bat-SARS-like coronavirus (BAT-SL-CoVZXC21 and BAT-SLCoVZC45).

2. Nitrogenase cofactor biosynthesis protein NifB [Betaproteobacteria bacterium HGWBetaproteobacteria-7] has a $37.04 \%$ identity with the ORF8 protein; it is involved in the process of biosynthesis of the iron-molybdenum cofactor found in the dinitrogenase enzyme of the nitrogenase complex in nitrogen-fixing microorganisms.

\subsection{Nucleocapsid phosphoprotein}

Its main function of this protein of the severe acute respiratory syndrome coronavirus (SARS$\mathrm{CoV}-1)$ is to package the positive strand viral genome RNA into a helical ribonucleocapsid (RNP) and plays a fundamental role during virion assembly through its contact with the viral genome and membrane protein $\mathrm{M}$, it also has an important role in enhancing the efficiency of subgenomic viral RNA transcription, in addition to viral replication. (5) As for molecular functions, the nucleoproteins are involved in RNA binding- the selective and non-covalently interaction with an RNA molecule or a portion of it. (5) The nucleoprotein is involved also in the following biological process: transcription (the transfer of genetic information from the RNA to mRNA by RNA-directed RNA polymerase).

In the case of Nucleocapsid phosphoprotein we found the following proteins with the help of the BLAST program to be related to this protein:

Nucleocapsid protein [Rhinolophus affinis coronavirus] and nucleocapsid protein [Bat Hp-betacoronavirus/Zhejiang2013], the first has $91.00 \%$ identity with the Nucleocapsid phosphoprotein, and the later has $54.62 \%$ identity (5). They are also known as $\mathrm{N}$ protein which is the most abundand in Rhinolophus affinis coronavirus and it is a highly immunogenic phosphoprotein that is normally very conserved (5). 


\subsection{ORF7b Protein}

The SARS-CoV ORF7b protein is predicted to be a 44-amino-acid, highly hydrophobic protein with no identified sequence homology to other viral or cellular proteins. Given the highly hydrophobic nature of ORF7b, it has been hypothesized that ORF7b is a transmembrane protein and possibly a viral structural protein (5). Although the expression of ORF7b in virus-infected cells has not been documented to date, ORF7b-specific antibodies have been detected in SARS patient convalescent-phase serum. Whereas the ORF7b coding region is highly conserved among sequenced SARS-CoV isolates, serial passage of SARS$\mathrm{CoV}$ in Vero cells resulted in a 29-nucleotide deletion in ORF7b, demonstrating that the putative ORF7b protein is not essential for viral replication in vitro. (37)

In the case of the ORF7b protein we found the following proteins to be similar with the help of the BLAST program:

1. nonstructural protein NS7b [Bat coronavirus RaTG13], which has a 97.67\% identity with the ORF7b protein, is an integral component of the virus' membrane (30) so it consists of the gene product and protein complexes having at least some part of their peptide sequence embedded in the hydrophobic region of the membrane. (38)

2. hypothetical protein CSA42_03345 [Gammaproteobacteria bacterium], which has a $50.00 \%$ identity with the ORF7b protein, is responsible for protein secretion and it secretes virulence factors from the cytosol of the bacteria into host cells or the host environment

3. protein 9 [Rhinolophus affinis coronavirus], which has an $81.40 \%$ identity with the ORF7b protein, is also an integral component of the membrane of the Rhinolophus affinis coronavirus.

\subsection{ORF7a Protein}

This protein of Human SARS coronavirus (SARS-CoV) (Severe acute respiratory syndrome coronavirus) is a non-structural one that is dispensable for virus replication in cell culture (39). It is involved in a series of Biological processes such as the modulation by virus of host G0/G1 transition checkpoint, which represents any viral process that modulates the frequency, rate or extent of the cell G0/G1 transition checkpoint and the suppression by virus of host tethering activity that means any process in which a virus stops, prevents or reduces the activity of host tetherin activity; tetherin that is also known as BST2 is an alpha interferoninducible cellular factor that impairs the deliverance of a multitude of enveloped viruses; by blocking tethering activity, a lot of viruses circumvent their antiviral effects. (16)

For the ORF7a protein, we found out (using BLAST) that it has a $32.32 \%$ similarity with the ORF9 protein(5). This protein has its gene that encodes it included within the Nucleocapsid phosphoprotein, therefore its characteristics and functions we have already discussed.

\section{6. "S" Spike Glycoprotein}

The "S" Spike glycoprotein of Human SARS coronavirus (SARS-CoV) (Severe acute respiratory syndrome coronavirus) is a surface trimmer glycoprotein that is located on the 
envelope of the virions. It is divided into two subunits S1 and S2 that have different responsibilities: the first one is in charge of host receptor-binding while the other one the membrane-fusion machinery. (5)

Spike protein S1: attaches the virion to the cell membrane by interacting with the host receptor, initiating the infection. Binding to human ACE2 and CLEC4M/DC-SIGNR receptors and internalization of the virus into the endosomes of the host cell induces conformational changes in the S glycoprotein. Proteolysis by cathepsin CTSL may unmask the fusion peptide of $\mathrm{S} 2$ and activate membranes fusion within endosomes. (19)

Spike protein S2': Acts as a viral fusion peptide which is unmasked following S2 cleavage occurring upon virus endocytosis. (40)

Spike protein S2: mediates fusion of the virion and cellular membranes by acting as a class I viral fusion protein. Under the current model, the protein has at least three conformational states: pre-fusion native state, pre-hairpin intermediate state, and post-fusion hairpin state. During viral and target cell membrane fusion, the coiled-coil regions (heptad repeats) assume a trimer-of-hairpins structure, positioning the fusion peptide in close proximity to the $\mathrm{C}$ terminal region of the ectodomain. The formation of this structure appears to drive the apposition and subsequent fusion of viral and target cell membranes. (41)

In the case of Spike glycoprotein we found the following proteins with the help of the BLAST program to be related to this protein:

1. Spike glycoprotein from Bat coronavirus 279/2005, which has $79.1 \%$ identity with SARS-CoV-2's spike glycoprotein and the same function.

2. ATP synthase subunit $\mathbf{b}$, which has $34.7 \%$ identity with the Spike glycoprotein. Component of the $F_{0}$ channel, it forms part of the peripheral stalk, linking $F_{1}$ to $F_{0}$. $\mathrm{F}_{1} \mathrm{~F}_{0}$ ATP synthase produces ATP from ADP in the presence of a proton or sodium gradient. F-type ATPases consist of two structural domains, $F_{1}$ containing the intramembranous catalytic core and $\mathrm{F}_{0}$ containing the membrane proton channel, linked together by a central stalk and a peripheral stalk. During catalysis, ATP synthesis in the catalytic domain of $F_{1}$ is coupled via a rotary mechanism of the central stalk subunits to proton translocation. (5)

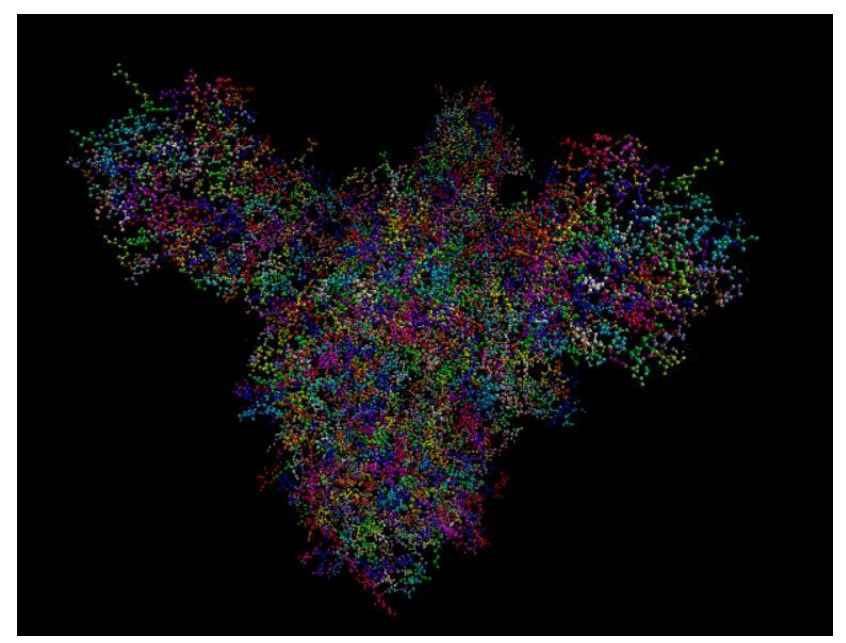

Picture 3 Protein seen in the VMD program, the colored parts are the Lysines and Glycines (42) (43) 


\section{7. "E" Envelope Protein}

This protein is found in small quantities within COVID-19 but one of the most striking functions displayed by the CoV E protein is ion channel (IC) activity. The CoV E protein self assembles in membranes forming pentameric protein-lipid pores that allow ion transport. (44) The $\mathrm{E}$ protein is the smallest of the major structural proteins, but also the most enigmatic. During the replication cycle, $\mathrm{E}$ is abundantly expressed inside the infected cell, but only a small portion is incorporated into the virion envelope. (5) The majority of the protein is localized at the site of intracellular trafficking, viz. the ER, Golgi, and ERGIC, where it participates in $\mathrm{CoV}$ assembly and budding. Recombinant CoVs have lacking $\mathrm{E}$ exhibit significantly reduced viral titers, crippled viral maturation, or yield propagation incompetent progeny, demonstrating the importance of $\mathrm{E}$ in virus production and maturation. (41) (45) Although it is not strictly necessary for viral replication it is definitely needed for pathogenesis.

In the case of Envelope protein we found the following proteins with the help of the BLAST program to be related to this protein:

1. Envelope small membrane protein (Bat coronavirus Rp3/2004) which has 100\% identity with our protein and the same function.

2. MscS Mechanosensitive ion channel found in Thioploca ingric, which has $46.7 \%$ identity with our protein. Its role is in transmembrane transport. (5)

3. Envelope small membrane protein from Murine coronavirus (strain A59) (MHVA59) (Murine hepatitis virus), which has 37\% identity with our protein. It is a component of the viral envelope that plays a central role in virus morphogenesis and assembly. (5) It is sufficient to form virus-like particles. It seems to be important for creating the membrane curvature needed to acquire the rounded, stable, and infectious particle phenotype. Acts as a viroporin, inducing the formation of hydrophilic pores in cellular membranes. It also induces apoptosis. (46).

\section{8. "M" Membrane Protein}

The $\mathrm{M}$ protein is the most abundant structural protein and defines the shape of the viral envelope. It is also regarded as the central organizer of $\mathrm{CoV}$ assembly, interacting with all other major coronaviral structural proteins. (5) Homotypic interactions between the $\mathrm{M}$ proteins are the major driving force behind virion envelope formation but, alone, is not sufficient for virion formation. Interaction of $S$ with $M$ is necessary for retention of $S$ in the ER-Golgi intermediate compartment (ERGIC)/Golgi complex and its incorporation into new virions, but dispensable for the assembly process. Binding of $\mathrm{M}$ to $\mathrm{N}$ stabilizes the nucleocapsid ( $\mathrm{N}$ protein-RNA complex), as well as the internal core of virions, and, ultimately, promotes completion of viral assembly. Together, $\mathrm{M}$ and $\mathrm{E}$ make up the viral envelope and their interaction is sufficient for the production and release of VLPs. (47)

In the case of the membrane protein we found the following proteins with the help of the BLAST program to be related to this protein:

1. Membrane protein from Bat coronavirus HKU3 (BtCoV) which has $97.1 \%$ identity with our protein and the same function. (5)

2. Membrane protein from Middle East respiratory syndrome-related coronavirus which has $48.6 \%$ identity with our protein. It is a component of the viral envelope that 
plays a central role in virus morphogenesis and assembly via its interactions with other viral proteins. (5)

3. Membrane protein from Transmissible gastroenteritis virus which has $32.5 \%$ identity with our protein and the same function: it is a component of the viral envelope that plays a central role in virus morphogenesis and assembly via its interactions with other viral proteins. (5)

\subsection{ORF3a Protein}

In SARS-CoV, orf3a, also called X1 or U274, is the largest of these ORFs and encodes a protein of 274 aa. It was predicted to contain an N-terminal signal peptide, followed by three transmembrane domains and a C-terminal cytoplasmic domain of approximately $150 \mathrm{aa}$. The 3a protein was localized to the plasma membrane and perinuclear regions of infected or transfected cells. (48)

The 3a protein is associated with virus particles produced following infection of Vero E6 or $\mathrm{CaCo} 2$ cells and assembles into virus-like particles when co-expressed with the $\mathrm{M}$ and $\mathrm{E}$ proteins in insect cells. It is O-glycosylated, interacts with the $\mathrm{M}, \mathrm{E}$, and S structural proteins and forms inter-chain disulfide linkages with the $S$ protein. (49)The protein is released in membranous structures from transiently expressing, as well as SARS-CoV-infected, cells and the deletion of its gene reduces virus growth. Convalescent sera from SARS patients contain antibodies to the $3 \mathrm{a}$ protein. In addition to a proposed structural role, the 3 a protein may also have regulatory functions. The ectopic expression of 3a induces apoptosis in Vero E6 cells and upregulates the expression of fibrinogen in A549 lung epithelial cells. Recently, the 3a protein has been shown to possess ion-channel activity selective for monovalent cations and was proposed to belong to the viroporin class of proteins. (50)

In the case of the ORF3a protein we found the following proteins with the help of the BLAST program to be related to this protein:

1. Protein 3 from Bat coronavirus Rp3/2004 (BtCoV/Rp3/2004) which has 68.6\% identity with ORF3a protein. It forms homotetrameric potassium sensitive ion channels (viroporin) and may modulate virus release. (5) Up-regulates expression of fibrinogen subunits FGA, FGB, and FGG in host lung epithelial cells. Induces apoptosis in cell culture. Downregulates the type 1 interferon receptor by inducing serine phosphorylation within the IFN alpha-receptor subunit 1 (IFNAR1) degradation motif and increasing IFNAR1 ubiquitination. (5)

\subsection{ORF6 Protein}

ORF6 encodes for a $\sim 7 \mathrm{kDa}$ protein with a hydrophobic $\mathrm{N}$-terminal and that has been suggested to have an N-endo-C-endo conformation. (5) Several groups have undertaken to characterize the protein product of the ORF6 gene and found that it interacts with the nsp8 protein from the SARS replicase complex, is able to increase infection titer during early infection at low multiplicity of infection, increase the rate of cellular gene synthesis, inhibit interferon production, and inhibit the nuclear translocation of STAT1 by interacting with karyopherin a2. (51)

Most recently, the ORF6 protein has been suggested to induce intracellular membrane rearrangements resulting in a vehicular population in the infected cell which could possibly serve some role in increasing replication. (16) 
Using BLAST we found out that ORF6 protein has an identity with Protein translocase subunit SecE from Antarcticibacterium sp. PAMC 28998. It is an essential subunit of the Sec protein translocation channel SecYEG. Clamps together the 2 halves of SecY. May contact the channel plug during translocation. (52)

\subsection{ORF10 Protein}

We tried to find ORF10's structure and function using BLAST but there was so significant result. So ORF10 protein is a SARS-CoV-2 particularity that needs further investigations.

\section{Conclusions}

The 2019-nCoV epidemic is still continuing. During the past months, imanes progress has been made in pathogen monitoring, identifying sources, basic etiology, clinical treatment, drug testing, and vaccine development. With the efforts of all countries affected by this outbreak, we hope that the epidemic will disappear soon. Future research on viral replication, pathogenesis, antiviral drugs, and other aspects of 2019-nCoV is desperately needed as it will contribute to the treatment and prevention of the virus. The high lethality outbreaks of viruses like SARS-CoV, MERS-CoV, H5N1, H7N9, Ebola, and the new 2019-nCoV should serve as an alarm to the world. We should try our best to reduce the probability of this viruses occurrence and effects by concentrating more on the investigation of animal etiology, normalizing the storage of high-risk pathogens, reducing direct contact with wild animals, maintaining the barriers between natural reservoirs and human society and completely eradicating wildlife trading will surely help. (9)

Furthermore, as we already pointed out the SARSCov2 is extremely similar with SARSCov1, so the therapeutics and vaccines used in 2003 can be equally effective for patients infected with the new COVID-19.

Also, we encourage people to respect the basic protective measures against this new coronavirus proposed by the World Health Organisation that include washing hands, avoiding close contact or spitting in public. 


\section{Bibliography}

1. Virus taxonomy: the database of the International Committee on Taxonomy of Viruses (ICTV). Lefkowitz, Elliot J and Dempsey, Donald $\mathrm{M}$ and Hendrickson, Robert Curtis and Orton, Richard J and Siddell, Stuart G and Smith, Donald B. s.I. : Oxford University Press, 2018, Vol. 46. D1.

2. National Institute of Alergy and Infectious Diseases. Coronaviruses. National Institute of Alergy and Infectious Diseases. [Interactiv] 18 February 2020. [Citat: 25 February 2020.] https://www.niaid.nih.gov/diseases-conditions/coronaviruses.

3. Coronaviruses: An Overview of Their Replication and Pathogenesis. Anthony R. Fehr, Stanley PerIman. Compton, United Kingdom : NCBI, 2015.

4. Medical Microbiology. 4th edition. David A.J. Tyrrell, Steven H. Myint. Galveston : Baron S, 1996.

5. UniProt: a worldwide hub of protein knowledge. Consortium, The UniProt. 2019, Nucleic Acids Res, 2019, Vol. 47. , pg. D507-515.

6. The molecular biology of coronaviruses\}. Masters, Paul S. s.I. : Elsevier, 2006, Vol. 66.

7. Viral Zone. Coronavirinae. Viral Zone. [Interactiv] SIB Swiss Institute of Bioinformatics, - - 2020. [Citat: 703 2020.] https://viralzone.expasy.org/785.

8. Learning from SARS: Preparing for the Next Disease Outbreak: Workshop Summary. Huang, Yanzhong. Washington (DC) : National Institute of Health, 2004. ISBN-10: 0-309-09154-3.

9. World Health Organisation. Cumulative Number of Reported Probable Cases of SARS. World Health Organisation. [Interactiv] 23 June 2003. [Citat: 31 January 2020.] https://www.who.int/csr/sars/country/2003_06_23/en/.

10. - . Update 49 - SARS case fatality ratio, incubation period. World Health Organisation. [Interactiv] 7 May 2003. [Citat: 31 January 2020.] https://www.who.int/csr/sarsarchive/2003_05_07a/en/.

11. Chan-Yeung M, Xu RH. SARS: epidemiology. Europe PMC. [Interactiv] ELIXIR Infrastructure, 1 November 2003. [Citat: 25 February 2020.] https://europepmc.org/article/med/15018127.

12. World Health Organisation. Epidemic curves - Severe Acute Respiratory Syndrome (SARS). World Health Organisation. [Interactiv] World Health Organisation, - - -. [Citat: 703 2020.] https://www.who.int/csr/sars/epicurve/epiindex/en/index1.html.

13. Altschul SF1, Gish W, Miller W, Myers EW, Lipman DJ. Basic local alignment search tool. PubMed.gov. [Interactiv] US National Library of Medicine, National Institutes of Health, 5 October 1990. [Citat: 28 February 2020.]

https://blast.ncbi.nlm.nih.gov/Blast.cgi?CMD=Web\&PAGE_TYPE=BlastDocs\&DOC_TYPE=References. 
14. European Center for Disease Prevention and Control. Q \& A on COVID-19. European Center for Disease Prevention and Control. [Interactiv] European Center for Disease Prevention and Control, 2020. [Citat: 9 May 2020.] https://www.ecdc.europa.eu/en/covid-19/questions-answers.

15. Organisation, World Health. Coronavirus disease (COVID-19) Pandemic. World Health Organisation. [Interactiv] World Health Organisation, 9 May 2020. [Citat: 9 May 2020.] https://www.who.int/emergencies/diseases/novel-coronavirus-2019.

16. Coronavirus Disease 2019 (COVID-19): A Perspective from China. Zu, Zi Yue, și alții, și alții. 2019.

17. Hui Li, Liang Liu, Dingyu Zhang, Jiuyang Xu, Huaping Dai, Nan Tang, Xiao Su, Bin Cao. SARS-CoV2 and viral sepsis: observations and hypotheses. TheLancet. [Interactiv] Elsevier Ltd, 9 May 2020. [Citat: 9 May 2020.] https://www.thelancet.com/pdfs/journals/lancet/PIIS0140-6736(20)30920X.pdf.

18. Hui Li, MD,Prof Liang Liu, MD Prof Dingyu Zhang, MD Jiuyang Xu, MD Prof Huaping Dai, MD Nan Tang, PhD Prof Xiao Su, PhD Prof Bin Cao, MD. SARS-CoV-2 and viral sepsis: observations and hypotheses. The Lancet. [Interactiv] The Lancet, 17 April 2020. [Citat: 23 April 202.] https://www.thelancet.com/journals/lancet/article/PIIS0140-6736(20)30920-X/fulltext.

19. The epidemic of 2019-novel-coronavirus (2019-nCoV) pneumonia and insights for emerging infectious diseases in the future. Jin-Yan Li, Zhi You,Qiong Wang,Zhi-Jian Zhou,Ye Qiu,Rui Luo,Xing-Yi Ge. s.I. : Elsevier, 2020, Vol. Microbes and Infection.

20. DrugBank. COVID-19 Information. DrugBank. [Interactiv] DrugBank, April 2020. [Citat: 24 April 2020.] https://www.drugbank.ca/covid-19.

21. A new antiviral drug Triazavirin: results of phase II clinical trial. Kiselev OI, Deeva EG, Mel'nikova TI, Kozeletskaia KN, Kiselev AS, Rusinov VL, Charushin VN, Chupakhin ON. 6, s.I. : PubMed, 2012, Vol. 56.

22. Nonstructural proteins NS7b and NS8 are likely to be phylogenetically associated with evolution of 2019-nCoV. MuhamadFahmia, YukihikoKubotab,Masahirolto. s.I. : Elsevier B.V., 2020, Vol. 81.

23. ORF1a-encoded replicase subunits are involved in the membrane association of the arterivirus replication complex. Van der Meer Y., van Tol H., Locker J.K., Snijder E.J. s.I. : J. Virol., 1998, Vol. 72:6689-6698.

24. Receptor recognition by the novel coronavirus from Wuhan: an analysis based on decade-long structural studies of SARS coronavirus. Wan Y., Shang J., Graham R., Baric R.S., Li F. s.I. : J. Virol., 2020, Vol. 94 doi: 10.1128/JVI.00127-20.

25. Structure of the SARS-CoV nsp12 polymerase bound to nsp7 and nsp8 co-factors. Kirchdoerfer R.N., Ward A.B. s.I. : Nat. Commun., 2019, Vol. 2019;10:2342.

26. Variant analysis of COVID-19 genomes. Koyama T., Platt D., Parida L. s.I. : Bull. World Health Organ., 2020, Vol. 10.2471/BLT.20.253591.

27. Quick Go. GO:0039520 induction by virus of host autophagy. Quick Go. [Interactiv] EMBL-EBI, 18 April 2019. [Citat: 4 February 2020.] https://www.ebi.ac.uk/QuickGO/term/GO:0039520. 
28. -. GO:0019079 viral genome replication. Quick Go. [Interactiv] EMBL-EBI, 2708 2018. [Citat: 5 February 2020.] https://www.ebi.ac.uk/QuickGO/term/GO:0019079.

29. - . GO:0019082 viral protein processing. Quck Go. [Interactiv] EMBL-EBI, 12 May 2017. [Citat: 5 February 2020.] https://www.ebi.ac.uk/QuickGO/term/GO:0019082.

30. UniProtKB . UniProtKB - U5WL93 (U5WL93_CVHSA). UniProt Consortium. [Interactiv] ELIXIR, 22 January 2014. [Citat: 18 March 2020.] https://www.uniprot.org/uniprot/U5WL93.

31. Biochemical and Structural Insights into the Mechanisms of SARS Coronavirus RNA Ribose 2'-OMethylation by nsp16/nsp10 Protein Complex. Yu Chen, Ceyang Su, Min Ke, Xu Jin, Lirong Xu, Zhou Zhang, Andong Wu, Ying Sun, Zhouning Yang, Po Tien, Tero Ahola, Yi Liang, Xinqi Liu, and Deyin Guo. https://doi.org/10.1371/journal.ppat.1002294, Wuhan : PLOS Pathogens, 2011.

32. SARS-Coronavirus Open Reading Frame-8b triggers intracellular stress pathways and activates NLRP3 inflammasomes. Shi C.S., Nabar N.R., Huang N.N., Kehrl J.H. s.I. : Cell Death Discov., 2019, Vol. 5:101.

33. Variant analysis of COVID-19 genomes. Koyama T., Platt D., Parida L. s.I. : Bull. World Health Organ., 2020, Vol. doi: 10.2471/BLT.20.253591.

34. SARS-CoV-2 and COVID-19: The most important research questions. Kit-San Yuen, Zi -Wei Ye, SinYee Fung, Chi-Ping Chan, Dong-Yan Jin. s.I. : Cell Biosci., 2020, Vol. 10: 40.

35. Quick Go. GO:0046718 viral entry into host cell. Quick Go. [Interactiv] EMBL-EBI, 26 January 2020. [Citat: 7 February 2020.] https://www.ebi.ac.uk/QuickGO/term/GO:0046718.

36. - . GO:0019062 virion attachment to host cell. Quck Go. [Interactiv] EMBL-EBI, 27 August 2018. [Citat: 7 February 2020.] https://www.ebi.ac.uk/QuickGO/term/GO:0019062.

37. The ORF7b protein of severe acute respiratory syndrome coronavirus (SARS-COV) is expressed in virus-infected cells and incorporated into SARS-CoV particles. Schaecher SR, Mackenzie JM, Pekosz A. s.I. : J Virol. , 2007, Vol. 81(2):718-31. .

38. Quick Go. Qucik Go. integral component of membrane. [Interactiv] EMBL-EBI, 2805 2015. [Citat: 20 March 2020.] https://www.ebi.ac.uk/QuickGO/term/GO:0016021.

39. An 81 base-pair deletion in SARS-COV-2 ORF7a identified from sentinel surveillance in Arizona. LaRinda A Holland, Emily A Kaelin, Rabia Maqsood, Bereket Estifanos, Lily I Wu, Arvind Varsani, Rolf U Halden, Brenda G Hogue, Matthew Scotch, Efrem S Lim. s.I. : Genome Reports, 2020.

40. "Genome composition and divergence of the novel coronavirus (2019-nCoV) originating in China,". al., A. Wu et. s.I. : Cell Host \& Microbe, 2020.

41. Remdesivir and chloroquine effectively inhibit the recently emerged novel coronavirus (2019$n$ CoV) in vitro. Wang, M., Cao, R., Zhang, L. et al. s.l. : Cell Res, 2020.

42. VMD - Visual Molecular Dynamics. Humphrey, W., Dalke, A. and Schulten. K. 33-38, s.I. : J. Molec. Graphics, 1996, Vol. Vol. 14 . 
43. The Protein Data Bank. Helen M. Berman, John Westbrook, Zukang Feng, Gary Gilliland, T. N. Bhat, Helge Weissig, Ilya N. Shindyalov, Philip E. Bourne. 1, s.l. : Nucleic Acids Research, 2000, Vol. Vol. 28.

44. The ORF7b Protein of Severe Acute Respiratory Syndrome Coronavirus (SARS-COV) Is Expressed in Virus-Infected Cells and Incorporated into SARS-CoV Particles. Scott R. Schaecher, Jason M.

Mackenzie and Andrew Pekosz. s.I. : J Virol. , 2006, Vol. 81(2): 718-731.

45. "A 193-amino acid fragment of the SARS coronavirus S protein efficiently binds angiotensinconverting enzyme 2.". Wong S.K., Li W., Moore M.J., Choe H., Farzan M. 2004, J. Biol. Chem. 279:3197-3201(2004), pg. 3197-3201.

46. Induction of apoptosis in murine coronavirus-infected cultured cells and demonstration of $E$ protein as an apoptosis inducer. An S1, Chen CJ, Yu X, Leibowitz JL, Makino S. Austin, Texas : J Virol., 1999, Vol. 73(9):7853-9.

47. "CD209L (L-SIGN) is a receptor for severe acute respiratory syndrome coronavirus.". Jeffers S.A., Tusell S.M., Gillim-Ross L., Hemmila E.M., Achenbach J.E., Babcock G.J., Thomas W.D. Jr., Thackray L.B., Young M.D., Mason R.J., Ambrosino D.M., Wentworth D.E., Demartini J.C., Holmes K.V. 2004, Proc. Natl. Acad. Sci. U.S.A. 101, pg. 15748-15753.

48. "Activation of the SARS coronavirus spike protein via sequential proteolytic cleavage at two distinct sites.". Belouzard S., Chu V.C., Whittaker G.R. 2009, Proc. Natl. Acad. Sci. U.S.A. 106, pg. 5871-5876.

49. SARS-CoV-2 and ORF3a: Non-Synonymous Mutations and Polyproline Regions. Elio Issa, Georgi Merhi, Balig Panossian, Tamara Salloum, Sima Tokajian. 2020.

50. SARS-CoV-2 and ORF3a: Nonsynonymous Mutations, Functional Domains, and Viral Pathogenesis. Issa E, Merhi G, Panossian B, Salloum T, Tokajian S. 2020.

51. "Severe acute respiratory syndrome coronavirus E protein transports calcium ions and activates the NLRP3 inflammasome.". Nieto-Torres J.L., Verdia-Baguena C., Jimenez-Guardeno J.M., ReglaNava J.A., Castano-Rodriguez C., Fernandez-Delgado R., Torres J., Aguilella V.M., Enjuanes L. 2015, Virology 485, pg. 330-339.

52. SARS-CoV-2 sensitive to type I interferon pretreatment. Kumari G. Lokugamage, Craig Schindewolf, Vineet D. Menachery. 2020.

53. A Novel Severe Acute Respiratory Syndrome Coronavirus Protein, U274, Is Transported to the Cell Surface and Undergoes Endocytosis. Yee-Joo Tan, Eileen Teng, Shuo Shen, Timothy H. P. Tan, PhuayYee Goh, Burtram C. Fielding, Eng-Eong Ooi, Hwee-Cheng Tan, Seng Gee Lim, and Wanjin Hong. s.I. : J Virol, 2004, Vol. 78.

54. A putative diacidic motif in the SARS-COV ORF6 protein influences its subcellular localization and suppression of expression of co-transfected expression constructs. Vithiagaran Gunalan, Ali Mirazimi and Yee-Joo Tan. s.I. : BioMed Central Ltd., 2011, Vol. 4. 
55. "Severe acute respiratory syndrome coronavirus envelope protein ion channel activity promotes virus fitness and pathogenesis.". Nieto-Torres J.L., DeDiego M.L., Verdia-Baguena C., JimenezGuardeno J.M., Regla-Nava J.A., Fernandez-Delgado R., Castano-Rodriguez C., Alcaraz A., Torres J., Aguilella V.M., Enjuanes L. 2014, PLoS Pathog. 10, pg. E1004077-E1004077.

56. Coronavirus envelope protein: current knowledge. Fielding, Dewald Schoeman \& Burtram C. 2019, Virol J 16, p. 69.

57. Severe Acute Respiratory Syndrome Coronavirus $3 a$ Protein Is Released in Membranous Structures from $3 a$ Protein-Expressing Cells and Infected Cells. Cheng Huang, Krishna Narayanan, Naoto Ito, C. J. Peters, Shinji Makino. s.I. : Journal of Virology, 2005, Vol. 80 (1) 210-217. 